\title{
Parametric Sensitivity Analysis of the WAVEWATCH III Model
}

\author{
Beng-Chun Lee ${ }^{1}$, Yang-Ming Fan ${ }^{2,}$, Laurence Zsu-Hsin Chuang ${ }^{3}$, and Chia Chuen Kao ${ }^{2}$ \\ ${ }^{1}$ Department of Environmental and Hazards-Resistant Design, Huafan University, Taipei 22301, Taiwan, ROC \\ ${ }^{2}$ Department of Hydraulic and Ocean Engineering, National Cheng Kung University, Tainan 701, Taiwan, ROC \\ ${ }^{3}$ Institute of Ocean Technology and Marine Affairs, National Cheng Kung University, Tainan 701, Taiwan, ROC
}

Received 23 November 2007, accepted 25 April 2008

\begin{abstract}
The parameters in numerical wave models need to be calibrated before a model can be applied to a specific region. In this study, we selected the 8 most important parameters from the source term of the WAVEWATCH III model and subjected them to sensitivity analysis to evaluate the sensitivity of the WAVEWATCH III model to the selected parameters to determine how many of these parameters should be considered for further discussion, and to justify the significance priority of each parameter. After ranking each parameter by sensitivity and assessing their cumulative impact, we adopted the ARS method to search for the optimal values of those parameters to which the WAVEWATCH III model is most sensitive by comparing modeling results with observed data at two data buoys off the coast of northeastern Taiwan; the goal being to find optimal parameter values for improved modeling of wave development. The procedure adopting optimal parameters in wave simulations did improve the accuracy of the WAVEWATCH III model in comparison to default runs based on field observations at two buoys.
\end{abstract}

Key words: Sensitivity analysis, Parameters, WAVEWATCH III model, Adaptive random search, Optimal values

Citation: Lee, B. C., Y. M. Fan, L. Z.-H. Chuang, and C. C. Kao, 2009: Parametric sensitivity analysis of the WAVEWATCH III model. Terr. Atmos. Ocean. Sci., 20, 425-432, doi: 10.3319/TAO.2008.04.25.01(Oc)

\section{INTRODUCTION}

For several decades, numerical wave models have been an integral part of weather prediction at weather forecast centers around the world. Major meteorological agencies now rely on so-called third-generation wave models such as WAM or WAVEWATCH III (WW3). In these models, all physical processes describing wave growth and decay are parameterized explicitly. Therefore, there are many empirical or tuning parameters in the formulas of models, and the setting of these parameters greatly influences the results of model simulation. Consequently, it is important to first determine the most influential parameters and then find their optimal values so as to improve model outcomes.

Sensitivity analysis (SA) (Saltelli et al. 2000) was created to deal simply with uncertainties in model parameters. Since its development, SA has been used widely in many fields. Gu and Li (2002) adopted SA to evaluate river temperature variations in response to changes in hydraulic and meteorological conditions. Vachaud and Chen (2002) an-

\footnotetext{
* Corresponding author

E-mail: yangming.fan@gmail.com
}

alyzed a large-scale hydrologic modeling problem using sensitivity theory. Nagai (2002) performed SA of a new atmosphere-soil-vegetation model setting on surface fluxes. Lamoureux et al. (2006) used SA to identify which factors had a greater effect on pond temperature. These effective applications of SA sparked our interest in adopting the SA method for our wave model parametric test in order to increase the accuracy of our model.

In this paper, we first analyze the WW3 to determine which of the model's many parameters most influence the model. We then conduct sensitivity analysis on these parameters to rank them by sensitivity; i.e., to which of the selected parameters is the model most sensitive. We then apply the adaptive random search (ARS) method (Törn and Zilinskas 1989) to determine the optimal values of the most important parameters. Finally, the results of our analysis are used to run the WW3; and a three-way comparison is made between actual buoy observations, our model, and a default run of WW3 (using default parameter settings) for coastal waters off northeastern Taiwan. 


\section{PARAMETERS SELECTED FROM THE WAVE MODEL}

\subsection{The WW3 Model}

WW3 (Tolman 2002) predicts the evolution in the twodimensional physical space $x$ and time $t$ of the wave action density spectrum $F$ of the wavenumber $k$ and direction $\theta$ as $F(k, \theta)$. This model was selected for our study because of its invariant characteristics with respect to the physics of wave growth and decay for variable water depths. The net source term $S$ consists of four parts: (1) a wind-wave interaction term, $S_{i n}$; (2) a nonlinear wave-wave interaction term, $S_{n l}$; (3) a dissipation (white cap) term, $S_{d s}$; and (4) a wave-bottom interaction term, $S_{b o t}$ (e.g., Shemdin et al. 1978):

$S=S_{i n}+S_{n l}+S_{d s}+S_{b o t}$

However, WW3 incorporates many empirical and tuning parameters that may or may not have importance from the standpoint of the physical processes that this model is meant to describe. As a result, to improve upon default runs of the model, it is important to select those parameters that are most influential over the model and then test the sensitivity of the model to these selected parameters. The parameters to be selected are defined in the formula of the source term.

\subsection{Description and Selection of Parameters}

\subsubsection{Nonlinear Wave-Wave Interactions Term}

Nonlinear wave-wave interactions can be modeled using discrete interaction approximation (DIA, Hasselmann et al. 1985). Nonlinear interactions occur between four wave components (quadruplets) with wavenumber vectors $\mathbf{k}_{1}$ through $\mathbf{k}_{4}$. For these quadruplets, the contribution, $\delta S_{n l}$, to the interaction for each discrete $\left(f_{r}, \theta\right)$ combination of the spectrum corresponding to $\mathbf{k}_{1}$ is calculated as:

$$
\begin{aligned}
\left(\begin{array}{l}
\delta S_{n l, 1} \\
\delta S_{n l, 3} \\
\delta S_{n l, 4}
\end{array}\right)= & D\left(\begin{array}{r}
-2 \\
1 \\
1
\end{array}\right) C g^{-4} f_{r, 1}^{11} \times\left\{F _ { 1 } ^ { 2 } \left[\frac{F_{3}}{\left(1+\lambda_{n l}\right)^{4}}\right.\right. \\
& \left.\left.+\frac{F_{4}}{\left(1-\lambda_{n l}\right)^{4}}\right]-\frac{2 F_{2} F_{3} F_{4}}{\left(1-\lambda_{n l}^{2}\right)^{4}}\right\}
\end{aligned}
$$

where $F_{1}=F\left(f_{r, 1}, \theta_{l}\right)$, etc., $\delta S_{n l, 1}=\delta S_{n l}\left(f_{r, l}, \theta_{l}\right)$, etc., $g$ is the acceleration of gravity, and $C$ is a proportionality constant. Nonlinear interactions are calculated by considering a limited number of combinations $\left(\lambda_{n l}, C\right)$. Default values in DIA for different input dissipation are quite different. Therefore, $C$ was one of the selected parameters here.

Formula (2) is developed for deep water, using the ap- propriate dispersion relation in resonance conditions. For shallow water, the wave expression is scaled by the factor $D$ :

$D=1+\frac{c_{1}}{\bar{k} d}\left(1-c_{2} \bar{k} d\right) e^{-c_{3} \bar{k} d}$

where $\bar{k} d=0.75 \hat{k} d, \hat{k}=(\overline{1 / \sqrt{k}})^{-2}, k$ is the wavenumber, $d$ is the mean water depth, and $c_{1}, c_{2}, c_{3}$ are default values for the constants (Hasselmann and Hasselmann 1985). The shallow water wave correction of Eq. (3) is valid for intermediate depths only. For this reason, the mean relative depth $\bar{k} d$ is not allowed to become less than 0.5 and it can be reset by the user in the input files of the model.

\subsubsection{Wind-Wave Interaction Term and Dissipation Term}

In this study, the input source term is adopted from Chalikov and Belevich (1993) and Chalikov (1995). However, during testing of a global implementation of WW3 including this source term (Tolman 2002), it was found that its swell dissipation due to opposing or weak winds was severely overestimated. To correct this deficiency, a filtered input source term is used. It is defined as:

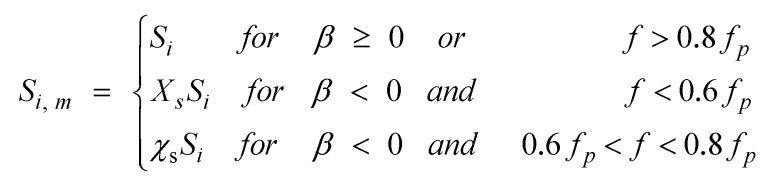

where $\beta$ is a nondimensional wind-wave interaction parameter, $f$ is the frequency, $f_{p}$ is the peak frequency of the wind sea computed from the input source term, $S_{i}$ is the input source term, and $X_{s}\left(0<X_{s}<1\right)$ is a reduction factor for $S_{i}$, which is applied to swell with negative $\beta$ only (defined by the user). $\chi_{\mathrm{s}}$ represents a linear reduction of $X_{s}$ with $f_{p}$ providing a smooth transition between the original and the reduced input. Therefore, $X_{s}$ is one of the selected parameters here.

Test results of these source terms in a global model implementation (Tolman 2002) suggested that the wind speed $u$ can be replaced by an effective wind speed $u_{e}$. In Tolman (2002), the following effective wind speed is used:

$$
\begin{aligned}
& \frac{u_{e}}{u}=\left(\frac{c_{0}}{1+C_{1}+C_{2}}\right)^{-1 / 2} \\
& C_{1}=c_{1} \tanh \left\{\max \left[0, f_{1}\left(S T-S T_{0}\right)\right]\right\} \\
& C_{2}=c_{2} \tanh \left\{\max \left[0, f_{2}\left(S T-S T_{0}\right)\right]\right\}
\end{aligned}
$$


$S T=\frac{h g}{u_{h}^{2}} \frac{T_{a}-T_{S}}{T_{0}}$

where $S T$ is a bulk stability parameter, $T_{a}, T_{s}$, and $T_{0}$ are the air, sea, and reference temperature, respectively, and $u_{h}$ is the wind speed at $h \mathrm{~m}$ height. Furthermore, $f_{1} \leq 0, c_{1}$ and $c_{2}$ have opposite signs and $f_{2}=f_{1} c_{1} / c_{2}$. The default parameter $c_{o}$ setting can be redefined by the user in the program input files.

\subsubsection{Wave-Bottom Interaction Term}

Wave-bottom interaction has to be considered in a shallow water environment. Here, using the notation of Tolman (1991), it can be written as:

$S_{b o t}(k, \theta)=2 \Gamma \frac{n-0.5}{g d} N(k, \theta)$

where $\Gamma$ is an empirical constant, which can be redefined by the model input files, and $n$ is the ratio of phase velocity to group velocity.

\subsubsection{The Spectral Shape of the Source Term}

Due to the maximum change in action density, $\Delta N_{m}$ is determined from a parametric change of action density $\Delta N_{p}$, and a filtered relative change $\Delta N_{r}$.

$$
\begin{aligned}
& \Delta N_{m}(k, \theta)=\min \left[\Delta N_{p}(k, \theta), \Delta N_{r}(k, \theta)\right] \\
& \Delta N_{p}(k, \theta)=X_{p} \frac{\alpha}{\pi} \frac{(2 \pi)^{4}}{g^{2}} \frac{1}{\sigma k^{3}} \\
& \Delta N_{r}(k, \theta)=X_{r} \max \left[N(k, \theta), N_{f}\right] \\
& N_{f}=\max \left\{\Delta N_{P}\left(k_{\max }, \theta\right), X_{f} \max _{\forall k, \theta}[N(k, \theta)]\right\}
\end{aligned}
$$

where $X_{p}, X_{r}$, and $X_{f}$ are user-defined parameters in the source term integration scheme, $\alpha$ is a PM (PiersonMoskowitz) energy level, and $k_{\max }$ is the maximum discrete wavenumber.

Summarizing the above analysis of the source term, the following parameters were selected to conduct sensitivity analyses and seek optimal initial values for the model: $c_{o}$ in $S_{i n,}$, representing a direct wind correction; $C$ in $S_{n l}$, being a proportionality constant; $\bar{k} d$ in $S_{n l}$, being the mean relative depth; $X_{s}$ in $S_{i n}$, being a reduction factor during opposing or weak winds; $\Gamma$ in $S_{b o t}$, being an empirical constant; $X_{p}, X_{r}$, and $X_{f}$, being the user-defined constants during simulated action density.

\section{SENSITIVITY ANALYSIS FOR THE PARAMETERS}

\subsection{Sensitivity Analysis and the Objective Function Evaluation Index}

Generally, SA is used to offer information on the accuracy of or errors in model results. It shows how model response results in slight changes to parameter values during simulation. Here, we propose a method by which SA can be used to analyze the sensitivity of the model to the selected parameters. These sensitivities are then used to rank the selected parameters.

To analyze the sensitivity or error of a time series from the results of the default run and the simulation run for model performance, this study adopts objective function (OBJ) as an evaluation index to determine the sensitivity of the parameters. In this section, the simulation run is defined as each parameter increases at a fixed ratio, for example, $\pm 10 \%, \pm 20 \%$, and $\pm 50 \%$, to the default value as an input in the model. Hence, there are six simulation runs for each parameter. The grid point of the model output is close to the buoy station used to gather actual observations. The final OBJ of each parameter is calculated using the averaged value of the difference in the model results between the default run and the simulation run after running the six simulations. The OBJ is defined as:

$$
\begin{aligned}
& \text { OBJ }=\sum_{i=1}^{N}\left[\left(O_{i}-M_{i}\right)^{2} \times W T_{i}\right] / N \\
& W T_{i}=\left(O_{i}+\bar{O}\right) /(2 \times \bar{O})
\end{aligned}
$$

where $O_{i}$ is the time series of the output of the default run, $M_{i}$ is the time series of the result of the simulation run, $N$ is the number of the time series, $W T_{i}$ is the weighting coefficient, and $\bar{O}$ is the average of the time series of the default run. The smaller the value of OBJ is, the lesser the error between the default run and the simulation run will be. In this paper, we use OBJ analysis to determine the sensitivity of each selected parameter. We also use the results of OBJ analysis in a latter section to discuss errors in significant wave height and mean wave period for both the winter monsoon and Typhoon Dujuan.

\subsection{Objective Function Analysis Using the Data of Taiwanese Waters}

In order to calculate sensitivity in the model, basic data for the period 1 November to 15 December 2003 (winter monsoon) was used to drive the model. In this case, the domain of the model covers longitude 100 to $145^{\circ} \mathrm{E}$ and latitude 0 to $45^{\circ} \mathrm{N}$, with a $0.5^{\circ}$ grid resolution in longitude and 
latitude (Fig. 1, inset map). The domain of the wind field data also covers the same region with a $0.5^{\circ}$ grid resolution at 1-h resolution. Two corresponding buoy stations (Longdong and Hualien) are also shown in Fig. 1.

The sensitivities of the eight parameters $\left(c_{o}, X_{p}, C, X_{s}, \Gamma\right.$, $\bar{k} d, X_{f}$, and $X_{r}$ ) selected for this study by OBJ for the two dedicated buoy stations are shown in Table 1 . The normalized OBJ of each parameter is shown in Fig. 2. The results reveal

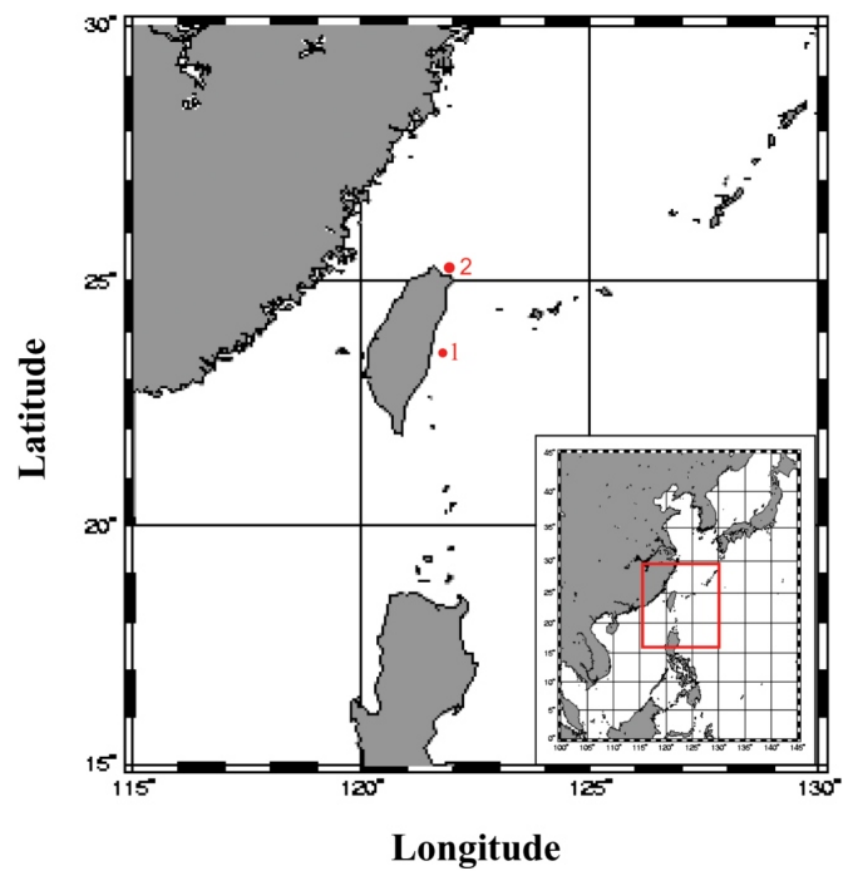

Fig. 1. Domain of model and buoy stations: 1, Hualien; 2, Longdong.

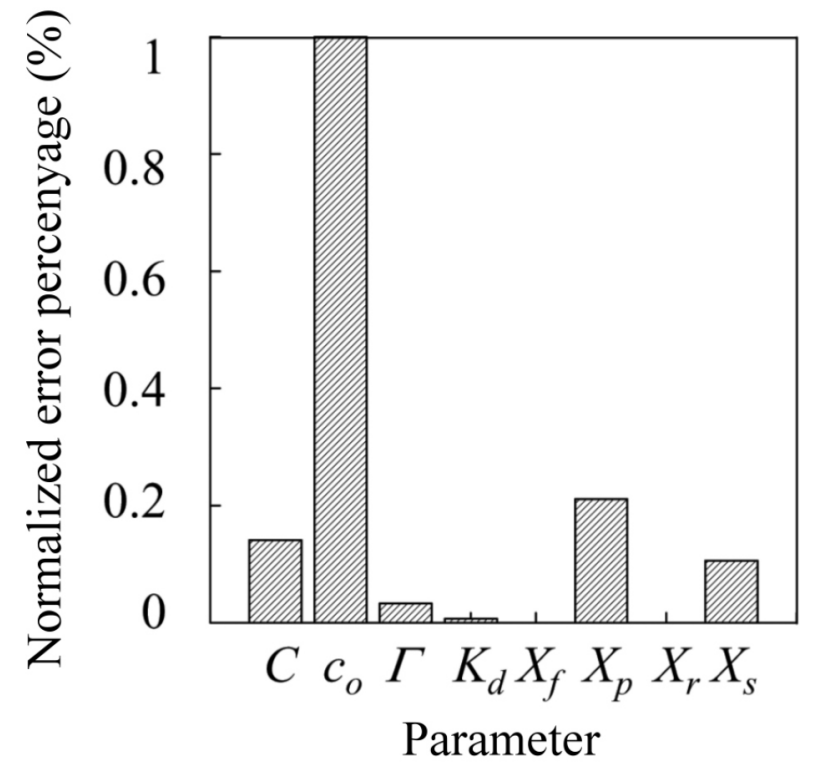

Fig. 2. Normalized OBJ of each parameter. that the most sensitive parameter is $c_{o}$, which represents a direct wind correction. The results also indicate that the sensitivity discrepancy for each parameter is large.

Using the sensitivities of the eight parameters shown in Table 1, we now determine the cumulative errors that occur in the model as a result of changing the selected parameters. We start by using the parameter to which the model is most sensitive, a direct wind speed correction $\left(c_{o}\right)$. In this analysis, $c_{o}$ changes, first, from the default value by a fixed ratio of $\pm 10 \%, \pm 20 \%$, and $\pm 50 \%$ for each model simulation. The other seven parameters are kept at their initial default values. We then sum the difference between the default run and the model outcome for each run containing a new value for $c_{0}$. The cumulative error for the six runs is 1.949 , which represents the sensitivity index in the model for the parameter $c_{0}$. Next, we perform the same analysis using the first two ranked parameters from Table 1 ; i.e., $c_{o}$ and $X_{p}$, which gave a cumulative error of 2.012. The final results of all selected parameters for the buoy stations at Longdong and Hualien are shown in Table 2.

The normalized cumulative averaged error is shown in Fig. 3. It is up to $99 \%$ for the four parameters $c_{o}, X_{p}, C$, and $X_{s}$. This means that model response is very sensitive to these four major parameters.

Table 1. OBJ and sensitivities of each parameter.

\begin{tabular}{ccc}
\hline Parameters & OBJ & Sensitivities \\
\hline$C$ & 0.686 & 3 \\
$c_{o}$ & 4.680 & 1 \\
$\Gamma$ & 0.036 & 5 \\
$\bar{k} d$ & 0.016 & 6 \\
$X_{f}$ & 0.000 & 7 \\
$X_{p}$ & 0.879 & 2 \\
$X_{r}$ & 0.000 & 7 \\
$X_{s}$ & 0.160 & 4 \\
\hline
\end{tabular}

Table 2. Cumulative error due to number of parameters.

\begin{tabular}{cc}
\hline Number of parameters & Cumulative error \\
\hline 1 & 1.949 \\
2 & 2.012 \\
3 & 2.041 \\
4 & 2.051 \\
5 & 2.054 \\
6 & 2.054 \\
\hline
\end{tabular}




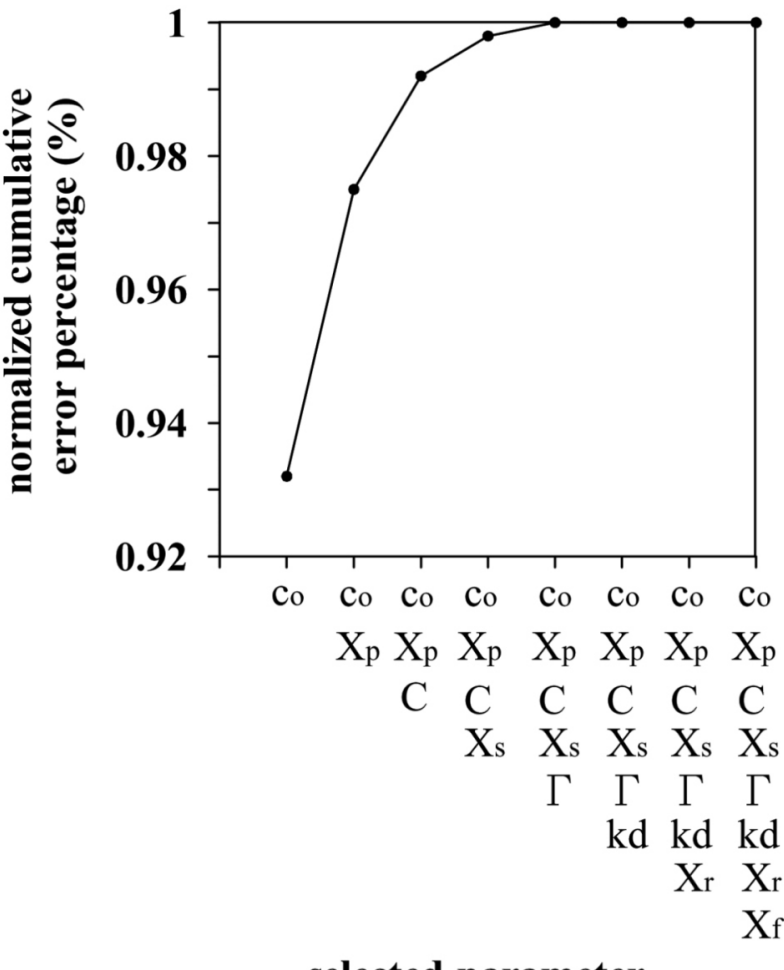

Fig. 3. Normalized cumulative errors of the parameters.

\section{SEARCHING FOR THE OPTIMAL VALUES OF SELECTED PARAMETERS}

\subsection{Adaptive Random Search}

Adaptive Random Search (ARS) is a tool (Törn and Zilinskas 1989) that can be used for obtaining regional optimal values using the macrocosm search method.

The object of the ARS is to find the optimal value of a model parameter; i.e., a parameter value that exhibits the least error between the simulation run and the default run. First, it is assumed that any default parameter is a center point along a linear axis with specific positive and negative units as the upper and lower boundaries (Fig. 4). The initial value of the model parameter is taken as one-tenth of the value between the lower boundary and the upper boundary; for example:

$$
\text { initial value }=(\text { upper boundary }- \text { lower boundary }) \times
$$

$$
\left[1+(10 \%)^{i}\right], i=1,2, \ldots 9
$$

The minimum error can be found between the simulation run and observation. At this point, the original default

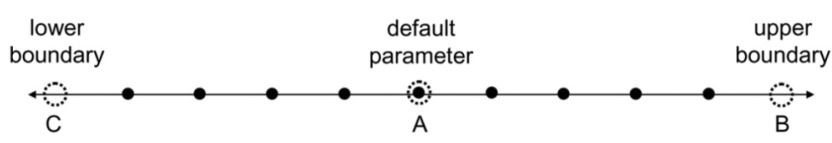

Fig. 4. Diagram of the searching range of the ARS method. value is replaced by this new value, which corresponds with the minimum error as the center point. Next, the same procedure again finds the optimal value, thus locating the second digital value of this new default value. Finally, by repeating this procedure several times, we can find the final, optimal value for this model parameter.

\subsection{Determining the Optimal Values of the Most Important Parameters}

In the case of Taiwanese waters, Taiwan's subtropical location in the western Pacific means that the northeast monsoon brings high or even huge waves to the northeastern coastal waters of Taiwan. By applying the ARS method, we hope to find the optimal values of the model parameters that best describe wave behavior during the winter monsoon. The final results of the optimal values for $c_{o}$, $X_{p}, C$, and $X_{s}$ (i.e., those parameters to which the model is most sensitive) in northeastern Taiwanese waters are listed in Table 3.

\section{VERIFICATION OF THE MODEL SIMULATION}

To verify the acquired optimal values of the model parameters (Table 3) we need to test the model in Taiwanese waters. This study adopts the winter monsoon and typhoon Dujuan for the model simulations. The experimental periods are from 20 to 29 February 2004 and from 30 August to 3 September 2003 for the winter monsoon and typhoon Dujuan, respectively, using wind-field data at 1-h resolution (Central Weather Bureau of Taiwan).

Figures 5 and 6 show the results of the time series of the model simulation for the Longdong buoy station in the winter monsoon. Figures 7 and 8 show the results for the Hualien buoy station in the winter monsoon. In the diagrams, the dots, solid line, and dashed line represent buoy observations, default simulation, and experimental simulation, respectively. The results from Figs. 5 and 7 reveal that wave height from the experimental simulation is more accurate than that of the default simulation. Whilst, the results from Figs. 6 and 8 indicate that the mean wave period from the experimental simulation is slightly better under the default simulation.

Table 3. Optimal parametric values for Taiwanese waters.

\begin{tabular}{ccc}
\hline Parameter & Default values & Tuned values \\
\hline$c_{o}$ & 1.4 & 1.25 \\
$C$ & $1.0 \times 10^{7}$ & $1.20 \times 10^{7}$ \\
$X_{p}$ & 0.15 & 0.25 \\
$X_{s}$ & 0.125 & 0.112 \\
\hline
\end{tabular}


Figures 9 and 10 show the results of the time series of the model simulation for the Longdong buoy station during typhoon Dujuan. Figures 11 and 12 show the results for the Hualien buoy station during typhoon Dujuan. In the diagrams, the dots, solid line, and dashed line represent buoy

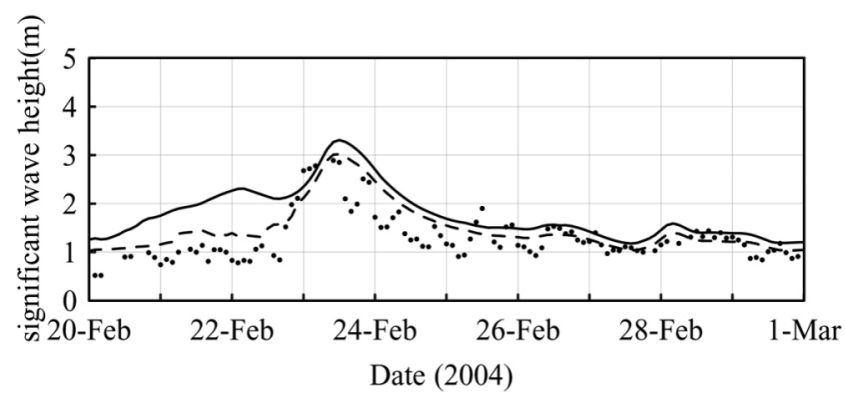

Fig. 5. $\mathrm{H}_{\mathrm{s}}$ time series at the Longdong data buoy in the winter monsoon.

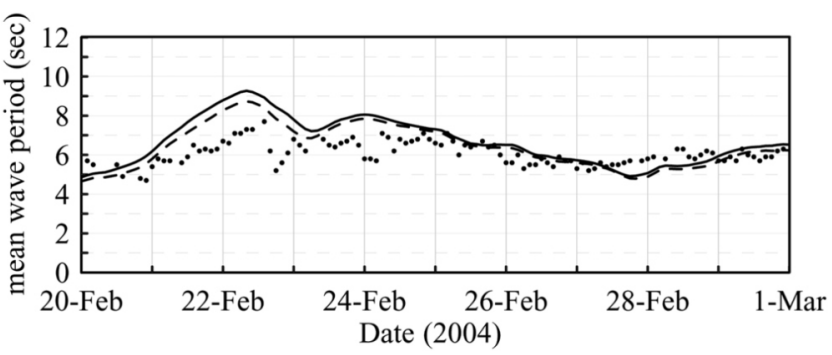

Fig. 6. $\mathrm{T}_{\mathrm{m}}$ time series at the Longdong data buoy in the winter monsoon.

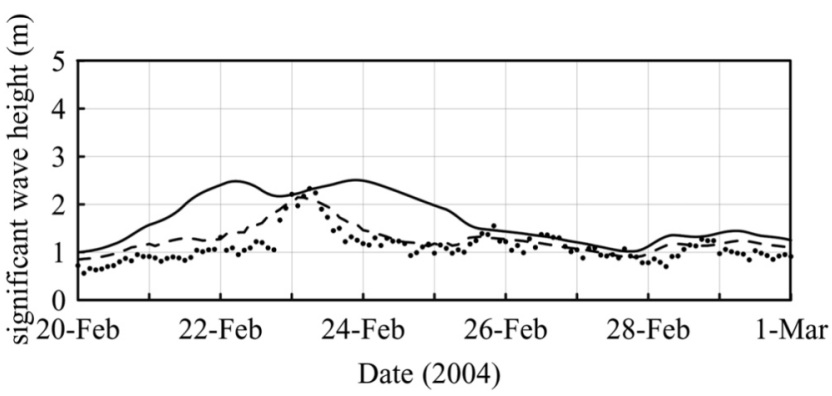

Fig. 7. $\mathrm{H}_{\mathrm{s}}$ time series at the Hualien data buoy in the winter monsoon.

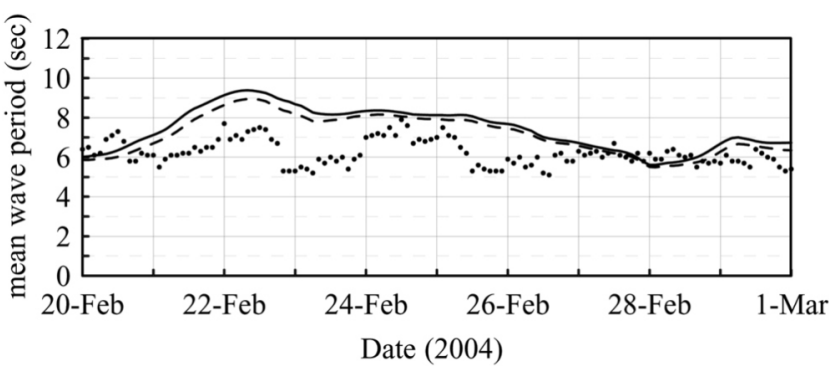

Fig. 8. $\mathrm{T}_{\mathrm{m}}$ time series at the Hualien data buoy in the winter monsoon. observations, default simulation, and experimental simulation, respectively. The results from Figs. 9 and 11 reveal wave height from the experimental simulation is more accurate than that of the default simulation. Similarly, the results from Figs. 10 and 12 indicate the mean wave period from the

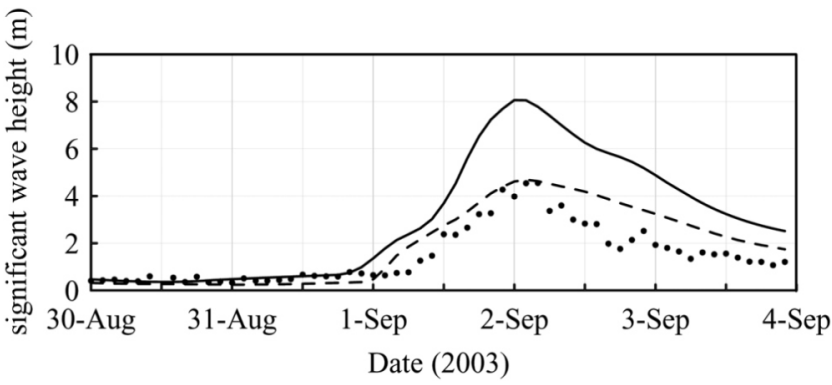

Fig. 9. $\mathrm{H}_{\mathrm{s}}$ time series at the Longdong data buoy in typhoon Dujuan.

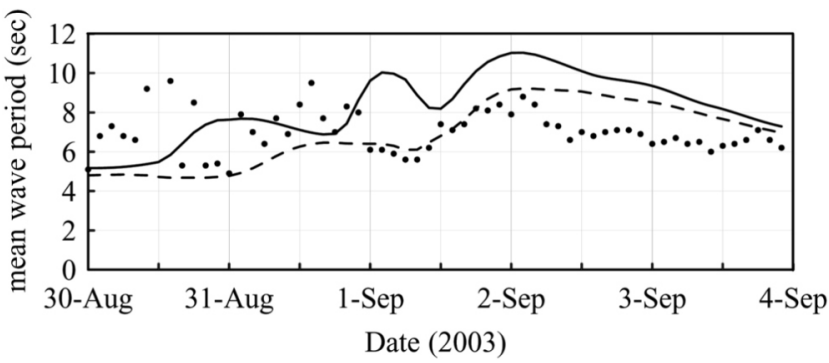

Fig. 10. $\mathrm{T}_{\mathrm{m}}$ time series at the Longdong data buoy in typhoon Dujuan.

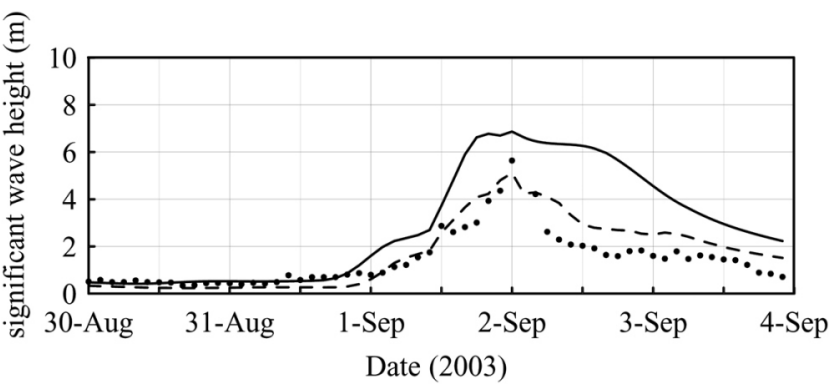

Fig. 11. $\mathrm{H}_{\mathrm{s}}$ time series at the Hualien data buoy in typhoon Dujuan.

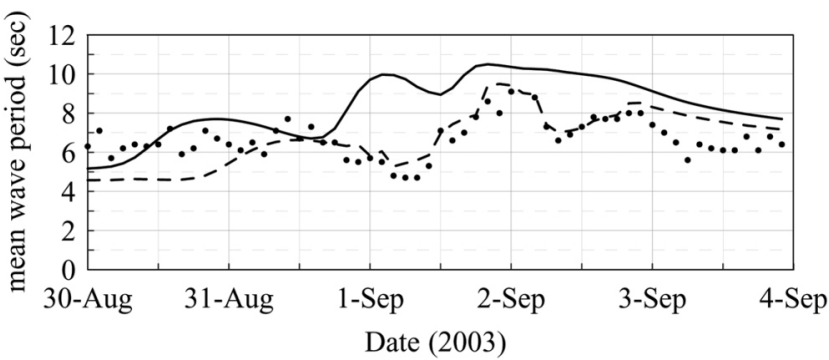

Fig. 12. $\mathrm{T}_{\mathrm{m}}$ time series at the Hualien data buoy in typhoon Dujuan. 
experimental simulation is more accurate than that of the default simulation.

The OBJ errors from the experimental and default simulations are listed in Tables 4 and 5. The error information and the model simulations indicate that the model is much improved for the experimental simulation of $\mathrm{H}_{\mathrm{s}}$, which adopted the optimal values of the parameter. In other words, this study improved accuracy in modeled wave height, but did not improve accuracy equally as well for the wave period. One reason for this might be that the wave spectrum overestimated the high frequency and underestimated low frequency during wave-growth calculations (Lee et al. 2006).

\section{CONCLUSIONS}

This paper provides a systemic way to search for optimal parameter values for improved modeling of wave development. The most influential parameters were selected from the source term of the WW3 and subject to sensitivity analysis and optimal value identification in order to improve the accuracy of the model over default runs (i.e., model runs using default parameter settings) for coastal waters off of northeastern Taiwan. Four parameters, the cumulative influence of which was given at $99 \%$ of errors, were found to be the most important factors in tuning the WW3. These four

Table 4. OBJ error of $\mathrm{H}_{\mathrm{s}}$ model simulations vs. specific buoy wave observation.

\begin{tabular}{lcc}
\hline Ptation & Darameter setting & Experimental simulation \\
\hline Longdong data buoy & 0.30 & 0.11 \\
Hualien data buoy & 0.37 & 0.06 \\
\hline
\end{tabular}

Table 5. OBJ error of $\mathrm{T}_{\mathrm{m}}$ model simulations vs. specific buoy wave observation.

\begin{tabular}{|c|c|c|}
\hline Station $\quad$ Parameter setting & Default simulation & Experimental simulation \\
\hline Longdong data buoy & 0.91 & 0.61 \\
\hline Hualien data buoy & 2.01 & 1.34 \\
\hline
\end{tabular}

parameters revealed by sensitivity analysis are: $c_{o}$ (a direct wind correction), $X_{p}$ (a constant of the PM wave spectrum), $C$ (a proportionality constant), and $X_{S}$ (a reduction factor during opposing or weak winds).

After ranking each parameter by model sensitivity, we adopted the ARS method to search for the optimal values of those four parameters to which the model is most sensitive. This was achieved by comparing modeling results with observed data at two buoys deployed at Longdong and Hualien off northeastern Taiwan.

The accuracy of wave simulations using optimal values for the selected parameters derived in this study was greatly improved over the default runs at the two data buoys. However, the same cannot be said for improved accuracy in wave period modeling. Here, slight improvement was observed for the simulation of Typhoon Dujuan but the winter monsoon result was less conclusive.

Acknowledgements The authors would like to thank the Central Weather Bureau and the Water Resources Agency for providing wind-field data and the observed wave data. Valuable discussions with Wen-Chin Lin are acknowledged.
The present study was supported by National Science Council grant NSC-93-2611-E-211-001.

\section{REFERENCES}

Chalikov, D. V., 1995: The parameterization of the wave boundary layer. J. Phys. Oceanogr., 25, 1333-1349, doi: 10.1175/1520-0485(1995)025<1333:TPOTWB >2.0.CO; 2. [Link]

Chalikov, D. V. and M. Y. Belevich, 1993: One-dimensional theory of the wave boundary layer. Bound.-Layer Meteor., 63, 65-96, doi: 10.1007/BF00705377. [Link]

Gu, R. R. and Y. Li, 2002: River temperature sensitivity to hydraulic and meteorological parameters. J. Environ. Manage., 66, 43-56, doi: 10.1006/jema.2002.0565. [Link]

Hasselmann, S. and K. Hasselmann, 1985: Computations and parameterizations of the nonlinear energy transfer in a gravity-wave spectrum, Part I: A new method for efficient computations of the exact nonlinear transfer integral. $J$. Phys. Oceanogr., 15, 1369-1377, doi: 10.1175/1520-0485 (1985)015<1369:CAPOTN>2.0.CO;2. [Link] 
Hasselmann, S., K. Hasselmann, J. H. Allender, and T. P. Barnett, 1985: Computations and parameterizations of the nonlinear energy transfer in a gravity-wave spectrum, Part II: parameterizations of the nonlinear energy transfer for application in wave models. J. Phys. Oceanogr., 15, 1378-1391, doi: 10.1175/1520-0485(1985)015<1378: CAPOTN $>2.0 . \mathrm{CO} ; 2$. [Link]

Lamoureux, J., T. R. Tierschb, and S. G. Hall, 2006: Sensitivity analysis of the pond heating and temperature regulation (PHATR) model. Aquac. Eng., 34, 117-130, doi: 10.1016/ j.aquaeng.2005.06.003. [Link]

Lee, B. C., H. Y. Cheng, C. C. Kao, and Y. M. Fan, 2006: On the estimation of wind wave of Taiwan water by using Swan Wave Model. The Proceedings of the 16th (2006) International Offshore and Polar Engineering Conference, 503507.

Nagai, H., 2002: Validation and sensitivity analysis of a new atmosphere-soil-vegetation model. J. Appl. Meteorol., 41, 160-176, doi: 10.1175/1520-0450(2002)041<0160: VASAOA $>2.0 . \mathrm{CO} ; 2$. [Link]

Saltelli, A., K. Chan, and E. M. Scott, 2000: Sensitivity analysis. John Wiley \& Sons LTD., 475 pp.
Shemdin, O., K. Hasselmann, S. V. Hsiao, and K. Heterich, 1978: Nonlinear and linear bottom interaction effects in shallow water. In Turbulent fluxes through the sea surface, wave dynamics and prediction, NATO Conference Series $\mathrm{V}, \mathbf{1}, 347-365$.

Tolman, H. L., 1991: A third-generation model for wind waves on slowly varying, unsteady and inhomogeneous depths and currents. J. Phys. Oceanogr., 21, 782-797, doi: 10.1175/1520-0485(1991)021<0782:ATGMFW>2.0.CO; 2. [Link]

Tolman, H. L., 2002: Testing of WAVEWATCH III version 2.22 in NCEP's NWW3 ocean wave model suite. NOAA/NWS/ NCEP/OMB Technology Note 214, 99 pp.

Tolman, H. L., 2002: User manual and system documentation of WAVEWATCH-III version 2.22. NOAA/NWS/NCEP/ MMAB Technical Note 222, 133 pp.

Törn, A. and A. Zilinskas, 1989: Global optimization. SpringerVerlag, New York, 255 pp.

Vachaud, G. and T. Chen, 2002: Sensitivity of computed values of water balance and nitrate leaching to within soil class variability of transport parameters. J. Hydrol., 264, 87100, doi: 10.1016/S0022-1694(02)00070-7. [Link] 